УДК 539.214

\title{
The Study of the Flow Stress During Superplastic Deformation Co-Ni-Nb Alloy
}

\author{
Aidyn E. Tussupzhanov ${ }^{a}$, Dosym Yerbolatuly, \\ Ludmila I. Kveglis ${ }^{\mathrm{b}}$ and Ivan V. Nemcev ${ }^{\mathrm{b}}$ \\ ${ }^{a}$ Sarsen Amanzholov East Kazakhstan State University \\ 34, 30 Gvardeiskoi divisii, Ust-Kamenogorsk, 070003, Kazakhstan \\ ${ }^{b}$ Siberian Federal University \\ 79 Svobodny, Krasnoyarsk, 660041, Russia
}

Received 13.03.2015, received in revised form 04.04.2015, accepted 10.06.2015

The article describes an experimental method for estimating the true flow stress at superplastic deformation. Studied changes of the true stress at superplastic deformation in optimally mode the precipitation-hardening alloy of cobalt-nickel-niobium. The microstructure of the alloy according to the extent of superplastic deformation.

Keywords: superplasticity, the true flow stress, microstructure, phase transformations.

DOI: 10.17516/1999-494X-2015-8-6-802-810.

\section{Исследование напряжения течения}

\section{при сверхпластической деформации}

Co-Ni-Nb-сплава

\section{А.Е. Тусупжанов ${ }^{a^{*}}$, Д. Ерболатулы Л.И. Квеглис ${ }^{\tilde{\sigma}}$ И.В. Немцев \\ ${ }^{a}$ Восточно-Казахстанский государственный университет имени С. Аманжолова Казахстан, 070003, Усть-Каменогорск, ул. 30 Гвардейской дивизии, 34 ${ }^{6}$ Сибирский федеральный университет Россия, 660041, Красноярск, пр. Свободный, 79}

В статье описана методика для оценки истинных напряжений течения экспериментальным методом при сверхпластической деформации. Проведены исследования изменения истинных

(c) Siberian Federal University. All rights reserved

* Corresponding author E-mail address: aidyn.tussupzhanov@mail.ru 
напряжений при сверхпластической деформации в оптимальном режиме дисперсионнотвердеющего сплава на кобальт-никель-ниобиевой основе. Исследована микроструктура сплава в зависимости от степени сверхпластической деформации.

Ключевые слова: сверхпластичность, истинные напряжения течения, микроструктура, фазовые превращения.

\section{Введение}

Основные преимущества использования эффекта сверхпластичности в технологии получения изделий связаны со значительным увеличением ресурса пластичности материалов. Кроме того, при формовке изделий из материала в состоянии сверхпластичности значительно снижаются усилия для деформации $[1,2]$. Поскольку при обработке металлов давлением в основном используется схема сжимающих напряжений, то в большинстве случаев привлекательно снижение усилий деформации. При этом снижается мощность требуемого деформирующего оборудования, нагрузка на инструмент и его износ [2]. Для создания технологии формовки деталей в режиме сверхпластичности (СП), помимо исследований взаимосвязи СП сплавов со структурно-фазовым состоянием и составом, необходимо установить особенности изменения деформирующих напряжений (прочности) в зависимости от степени деформации, температуры и скорости нагружения. В литературе нами не выявлено метода, который мог бы точно оценивать истинные напряжения течения при сверхпластичности. В связи с этим в настоящей работе для оценки истинных напряжений течения иследовали эволюцию структурнофазового состояния сплава $\mathrm{Co}-\mathrm{Ni}-\mathrm{Nb}$ в процессе сверхпластической деформации и особенности изменения напряжений течения.

\section{Образцы и методы исследования}

В качестве материала исследования выбран 67КН5Б $(67 \%-\mathrm{Co}, 28 \%-\mathrm{Ni}, 5 \%-\mathrm{Nb})$. Сплав $\mathrm{Co}-\mathrm{Ni}-\mathrm{Nb}$ относится к дисперсионно-твердеющим аустенитным сплавам и используется в промышленности. Выбор обоснован также и тем, что сплав проявляет СП свойства при определенной скорости и температуре деформации [3]. Сплав Co-Ni-Nb после закалки находится в состоянии $\gamma$-твердого раствора с ГЦК-решеткой и упрочняется при деформации или при старении за счет выделения избыточных вторичных фаз [3].

Деформационная обработка (прокатка) шла на самодельном двухвалковом прокатном стане. Закалку образцов от $950{ }^{\circ} \mathrm{C}$ проводили в трубчатых печах типа СУОЛ-4. Использовали кварцевые трубки, где создавался вакуум с давлением $10^{-3} \mathrm{MПа.} \mathrm{Сверхпластическую} \mathrm{деформацию}$ (СПД) осуществляли на установке для одноосного растяжения $1246 \mathrm{P}-2 / 2300$ в вакууме $10^{-6} \mathrm{MПа}$ с нагревом рабочей камеры до $870{ }^{\circ} \mathrm{C}$. Микроструктурные исследования образцов проводили на электронно-зондовом микроскопе S-3400N HITACHI и HITACHI TM-3000 в растровом режиме вторичных электронов, а также в режиме рентгеновского флуоресцентного микроанализа.

\section{Результаты и их обсуждение}

Ранее в работе [3] нами установлен способ обработки и оптимальный режим перевода аустенитного дисперсионно-твердеющего сплава $\mathrm{Co}-\mathrm{Ni}-\mathrm{Nb}$ в состояние сверхпластичности. Сверхпластичность достигалась после следующей термомеханической обработки: закалка от 
$950{ }^{\circ} \mathrm{C}(10$ мин $)+$ прокатка на $90 \%+$ СПД при $\mathrm{T}=870 \mathrm{~K}$ и $\varepsilon=0,72 \cdot 10^{-3} \mathrm{c}^{-1}[3]$. В результате величина относительного удлинения достигала значения $\delta=1140 \%$. Установлено влияние температуры выдержки перед закалкой и степени прокатки на микроструктуру и сверхпластическое свойство сплава. Обнаружено выделение ГПУ-фазы при отжиге и сверхпластической деформации прокатанного сплава Co-Ni-Nb. Установлены тип и параметры решетки, химический состав этой фазы, морфология и размеры её частиц, интервалы температур выделения, а также влияние фазы на СП и микротвердость сплава [3].

В данной работе исследованы изменения микроструктуры сплава Co-Ni-Nb в оптимальном режиме сверхпластической деформации и дана оценка истинных напряжений течения по специально разработанной методике. Метод основан на фотосъемке образца во время его высокотемпературного растяжения. Фотосъемка производились через каждые 13 минуты в зависимости от скорости движения захвата и ресурса пластичности материала; если ресурс пластичности материала небольшой, то, соответственно, интервал съемки уменьшаем.

В стандартной методике напряжение деформируемых образцов определяется по формуле

$$
\sigma_{i}=\frac{P_{i}}{F_{i}},
$$

где $P_{i}$ - усилие, приложенное к образцу в каждый момент измерения, определяется по первичной диаграмме растяжения; $F_{i}$ - площадь поперечного сечения образца.

Расчетным способом «истинные» напряжения СПД оцениваются $[2,4]$ с помощью следующей формулы:

$$
S_{\text {расч. }}=\frac{P_{i}}{F_{i}}=\frac{P_{i}}{F_{\text {o }}}(1+\varepsilon),
$$

где $\varepsilon=\Delta l / l_{0}-$ относительная деформация образца.

Формула не учитывает изменение площади поперечного сечения образца при сверхпластической деформации. Известно, что во время пластической деформации образцов площадь сечения образца уменьшается с увеличением степени деформации. В данной работе оценка площади сечения образцов производилась в режиме реального времени с помощью фотографирования шейки.

Поскольку формула (2) не учитывает изменение площади поперечного сечения, то величину поперечного сечения для цилиндрических образцов в измеряемый момент времени находят по формуле $F_{i}=\frac{\pi d_{i}^{2}}{4}$.

В случае плоских образцов мы предлагаем следующий способ определения $F_{i}$. Для плоского образца сечение равно произведению толщины и ширины $\mathrm{F}_{i}=a_{i} \cdot b_{i}$, где $a_{\mathrm{i}}-$ толщина и $b_{i}$ - ширина образца. Величину $b_{i}$ можно измерить по фотоснимкам (рис. 1). Измерения $b_{i}$ на фотоснимке мы проводили на самых тонких участках. Значения $a_{\mathrm{i}}$ для плоских образцов по фотоснимкам измерить не представляется возможным, поскольку съемка осуществлялась только с поверхности образца. В связи с этим на основании предположения, что толщина образца в ходе деформации изменяется по той же зависимости, что и его ширина, нами предложено следующее соотношение, связывающее эти параметры: 


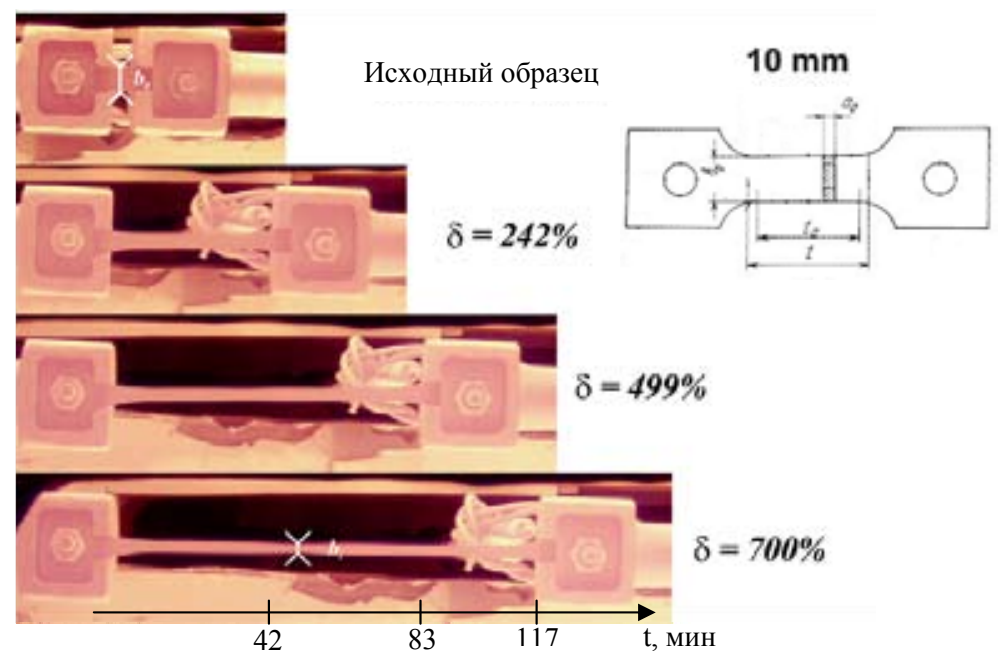

Рис. 1. Снимки образца во время сверхпластической деформации в установке для растяжения при температуре $\mathrm{T}=1143 \mathrm{~K}$ и скорости растяжения $\varepsilon=0,72 \cdot 10^{-3} \mathrm{c}^{-1}$

$$
a_{i}=\frac{b_{i} \cdot a_{0}}{b_{0}} .
$$

Справедливость этого соотношения доказана многократным измерением параметров $a_{0}$, $b_{0}, a_{i}$ и $b_{i}$

По нашему мнению, известный расчетный способ (2) определения истинных напряжений при СПД сплавов недостаточно достоверно описывает изменение истинных напряжений. Результаты экспериментов показывают, что все кривые напряжений, полученные тремя разными методами, совпадают только в начальный момент деформации, в пределах действия закона Гука (рис. 2 , кривая $1,2,3)$. Условные напряжения $\left(S_{\text {conv.s }}\right)$ вначале возрастают (сплав упрочняется), достигают максимума, затем наблюдается резкое уменьшение деформирующих напряжений с плавным переходом на пологий участок разупрочнения, продолжающийся вплоть до разрыва образца, что соответствует стандартному характеру кривой нагружения (рис. 2, кривая $1, S_{\text {conv.s }}$ [5].

Кривые «истинных» напряжений СП сплавов, полученные на основе расчета по формуле (2) $S_{\text {engin.s. }}$, в большинстве случаев повторяют форму зависимости условных напряжений. Однако в отличие от условных уровень расчетных «истинных» напряжений значительно выше (рис. 2, кривая 2, $S_{\text {engin.s. }}$ ).

На рис. 2 представлены графики изменения напряжения течения сплава в процессе СПД, построенные по данным, полученным: из первичной диаграммы растяжения (кривая $1, S_{c o n v . s}$ ),

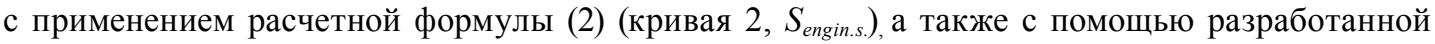
в настоящей работе экспериментальной методики - фотосъемки (кривая $3, S_{\text {expers.) }}$ в процессе растяжения. Кривые истинных напряжений, полученные сравнением фотографий, сделанных в процессе растяжения при фиксированных значениях времени, показывают, что деформация изменяется неравномерно. При этом установлено, что уровень истинных напряжений, полученных методом фотосъемки (кривая 3), при малых степенях деформации занимает промежу-

$$
-805-
$$




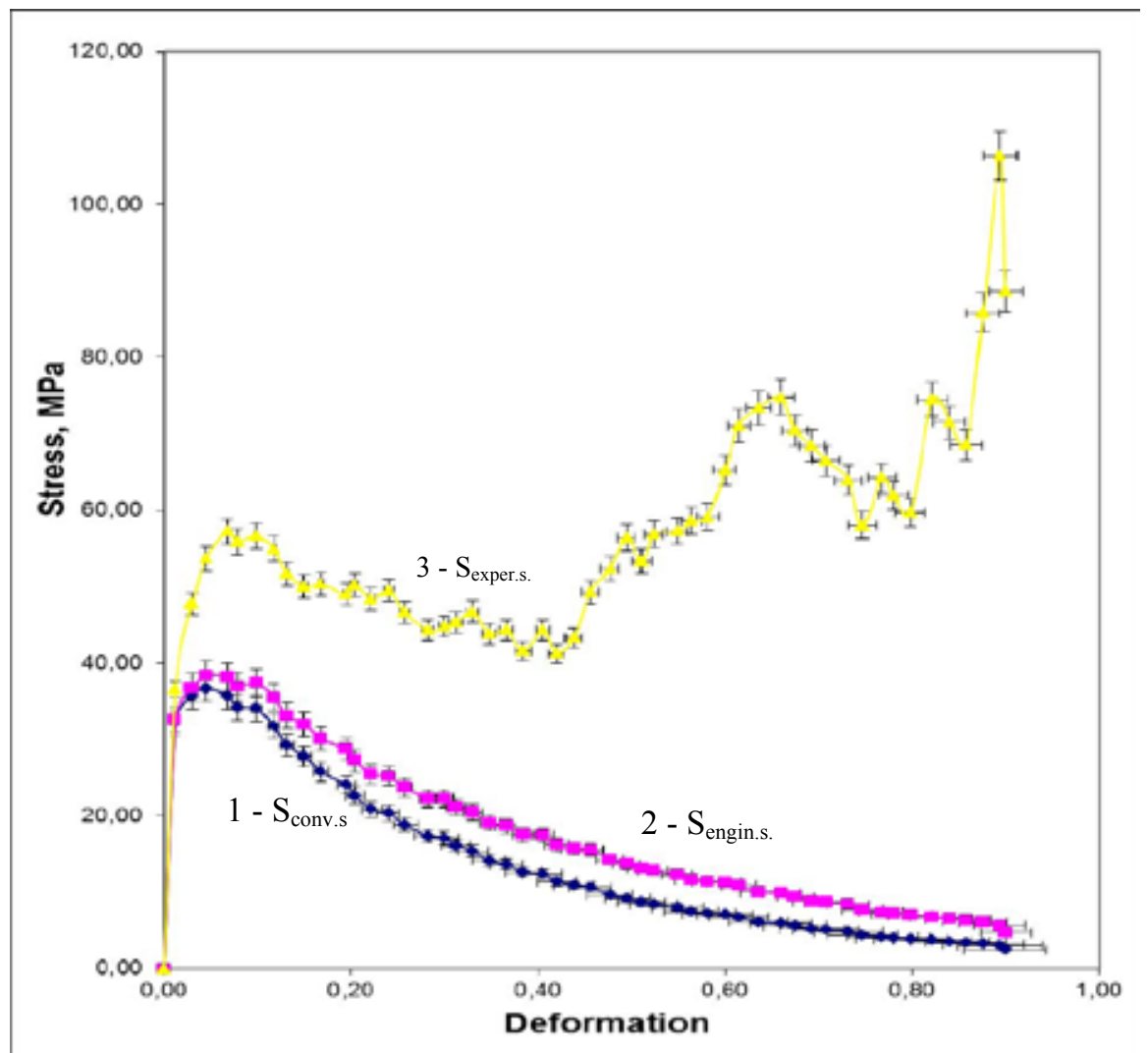

Рис. 2. График изменения условных и истинных напряжений течения сплава Co-Ni-Nb в процессе СПД при Т $=1143 \mathrm{~K}$ и $\varepsilon=0,72 \cdot 10^{-3} \mathrm{c}^{-1}: S_{\text {conv.s }}-$ условные напряжения, полученные на самописце; $S_{\text {engin.s. }}-$ истинные напряжения, полученные расчетным методом; $S_{\text {expers. }}-$ истинные напряжения, полученные фотометодом

точное значение между $S_{\text {conv.s }}$ (кривая 1) и $S_{\text {engin.s. }}$ (кривая 2) (рис. 2). При более высоких степенях деформации кривые «истинных» напряжений, полученные методом фотосъемки при фиксированном времени, показывают более высокие значения (рис. 2, кривая $3, S_{\text {exper.s. }}$ ). Таким образом, «истинные» напряжения оказываются значительно выше таковых, полученных расчетным методом по формуле (2).

На кривой $3\left(S_{\text {exper.s. }}\right)$ видны несколько максимумов. Первый максимум характеризует истинный предел прочности материала, который равен 57,18 МПа (табл. 1). По нашему предположению, второй максимум (74,79 МПа) получился вследствие образования «шейки», которая в процессе растяжения самоупрочняется и перестает развиваться. В результате удлинение образца происходит за счет других участков. В дальнейшем идет спад истинных напряжений течения до значений 58,04 МПа с некоторой стабилизацией. Затем характер увеличения напряжения и его спад при дальнейшем растяжении повторяется несколько раз. Также из графика видно, что в дальнейшем идет резкое повышение истинных напряжений с образованием последнего максимума (106,40 МПа) и затем наступает разрыв образца.

При СПД сплава Co-Ni-Nb при оптимальных условиях [6] относительное удлинение $\delta$ показывает максимальное значение 1140 \%. При этом истинное напряжение после начального -

$$
-806-
$$


максимума повышается до значения второго максимума 74,79 МПа и уменьшается. У данного образца разрыв произошел с образованием шейки, а при определении ширины образца методом фотосъемки замеры производят в наиболее узком участке. В процессе нагружения происходит уменьшение шейки разрыва, поэтому $S_{\text {expers. }}$ (кривая 3 , рис. 2 ) увеличивается до значений 106,40 МПа (табл. 1). Как показано в работе [3], вихревой характер пластического течения в шейке и возникновение дисклинаций в деформируемом материале обусловливают сильное деформационное упрочнение, которое блокирует поперечные сдвиги и вызывает в центре шейки нарушение сплошности материала.

Полученная зависимость напряжение-деформация имеет характер, описанный в работе [7], где показано, что существует критический поток энергии, при котором система скачкообразно меняет зависимость поглощения энергии от величины внешнего воздействия. Такие критические потоки энергии, получаемые образцом при его нагружении, могут соответствовать максимумам на кривой нагружения. Максимумы на кривой нагружения соответствуют изменениям внутренней энергии вещества, в результате чего происходят структурно-фазовые превращения [3]. На рис. 3 приведены зависимости неравновесного термодинамического потенциала Гиббса от молярного объема $F(\vartheta)$ [3].

Считаем, что каждый тип деформационного дефекта, как солитон кривизны определенного масштабного уровня, характеризуется собственным локальным минимумам. В сплавах на деформационных дефектах происходит зарождение новых фаз. Прерывистый распад в сплавах связан с локальной кривизной в границах зерен. В работе [8] показано, что после СПД, как и по-

Таблица 1. Механические характеристики сплава Co-Ni-Nb при СПД в оптимальных условиях

\begin{tabular}{|c|c|c|c|c|c|c|c|}
\hline$T,{ }^{\circ} \mathrm{C}$ & $S_{\text {conv.s, }} \mathrm{MPa}$ & $S_{\text {engin.s. }} \mathrm{MPa}$ & $\begin{array}{c}S_{\text {exper.s. }} \mathrm{MPa} \\
\text { (первый max.) }\end{array}$ & $\begin{array}{c}S_{\text {exper.s. }}, \mathrm{MPa} \\
\text { (второй max.) }\end{array}$ & $\begin{array}{c}S_{\text {exper.s. }} \text { (пРа } \\
\text { (посл. max.) }\end{array}$ & $\Psi, \%$ & $\delta, \%$ \\
\hline 870 & 34,23 & 38,37 & 57,18 & 74,79 & 106,40 & 97 & 1140 \\
\hline
\end{tabular}

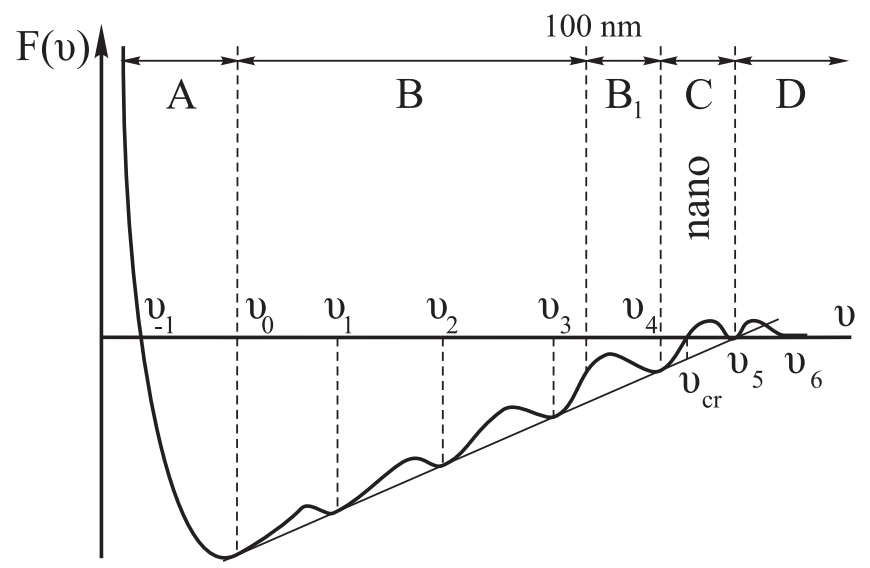

Рис. 3. Зависимость термодинамического потенциала Гиббса $F(\vartheta)$ от молярного объема $\vartheta$ с учетом локальных зон гидростатического растяжения различного масштаба, в которых возникают дефектные структуры:А-гидростатическоесжатие; В-мезосубструктурыразличныхмасштабов; $\mathrm{B}_{1}-$ наноразмерные структуры; C - наноструктурные состояния; D - возникновение пористости и разрушения [3]

$$
-807-
$$




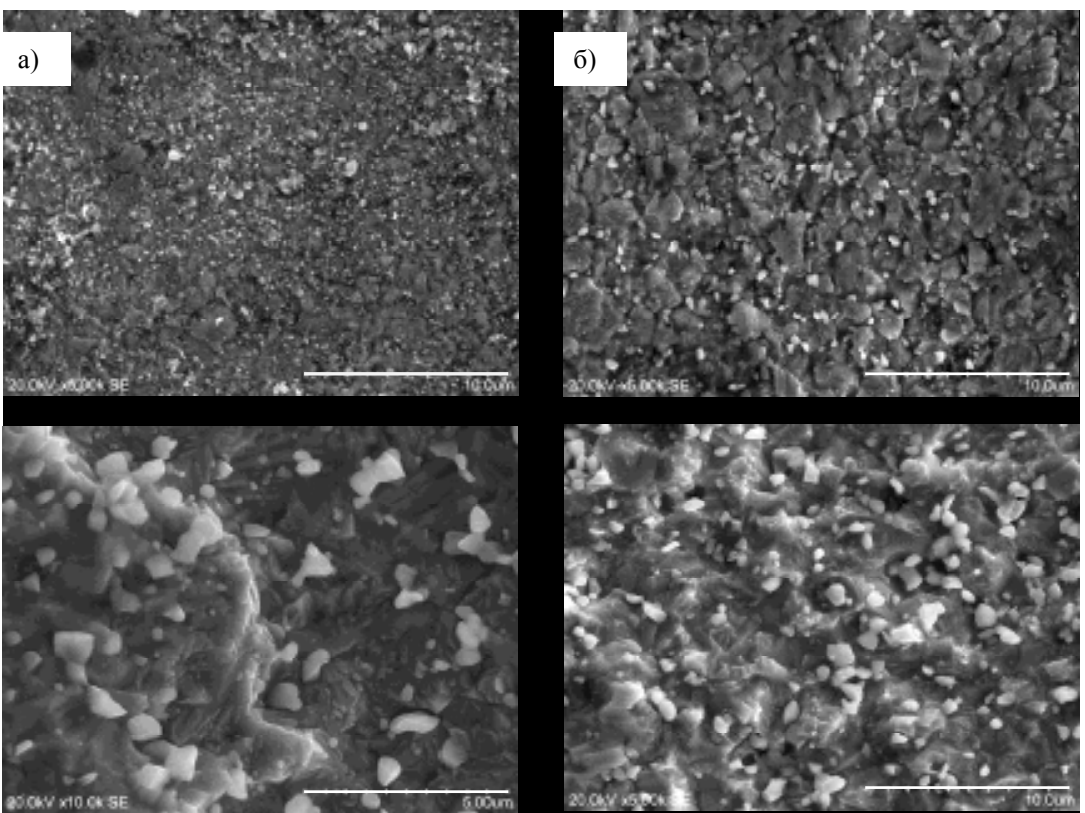

Рис. 4. Микроструктура сплава $\mathrm{Co}-\mathrm{Ni}-\mathrm{Nb}$ после различных степеней сверхпластической деформации при оптимальном режиме: $a$ - до сверхпластической деформаций; $b$ - растянут на $22 \%$; $c$ растянут на $530 \%$; $d$-растянут на $1050 \%$

сле отжига, в интервале температур СП в предварительно прокатанном на 90 \% сплаве Co-Ni$\mathrm{Nb}$ формируется двухфазная структура, состоящей из $\gamma$-фазы на основе однородного твердого раствора $\mathrm{Nb}$ в Co-Ni-матрице, имеющего ГЦК, с параметром, $a=0,356$ нм, и из п-фазы - твердый раствор замещения состава $(\mathrm{Co}, \mathrm{Ni}, \mathrm{Nb})$, имеющий ГПУ решетку с параметрами $a=5,62 \AA$, $c=7,90 \AA$ (рис. 4).

Установлено, что перед началом сверхпластической деформации в оптимальном режиме [6] происходит выделение вторичной фазы сферической формы размером $\approx 1$ мкм (рис. $4 a$ ). Из pис. $43 a, b$ видно, что выделение зерен вторичной фазы идет преимущественно по границам зерен, но процесс выделения также осуществляется и в теле зерен матрицы. При проведении дальнейшей сверхпластической деформации с большими степенями деформации идет рост вторичных зерен до 2 и более микрон и их коагуляция (рис. $4 b, d$ ). О коагуляции можно судить, по форме зерен, которые потеряли сферическую форму после сверхпластической деформации.

\section{Заключение}

С применением разработанной методики определения истинных напряжений установлена стадийность СПД. Достоверность полученных данных подтверждается хорошим согласием используемых в методике экспериментальных и расчетных данных, полученных при измерении образцов до и после разрушения.

В результате впервые установлен эффект аномального повышения истинных напряжений течения в предразрушительном этапе деформации, наиболее выраженный при оптимальных режимах СП. Несмотря на постоянство скорости движения захвата машины, с увеличением степени деформации происходит плавное снижение скорости деформации, свидетельствую- 
щее об упрочнении. Другими словами, наблюдается повышение напряжений с течением времени. Это противоречит данным работы [9], посвященной изучению кривых истинных напряжений течения сплавов эвтектоида алюминий-цинк, эвтектики олово-свинец и др. Авторами было обнаружено, что при некоторых температурах и степенях растяжения сопротивление деформированию снижается до аномально низких значений (меньше 0,25 МПа). Такое поведение металлов и сплавов было названо истинной сверхпластичностью [10]. Поэтому обнаруженный в настоящей работе эффект упрочнения сплавов при СПД действительно можно назвать аномальным, и для объяснения его требуются дальнейшие исследования с детальным изучением изменения структурно-фазового состояния образцов при деформации.

На основе сопоставления полученных кривых напряжений с фотоснимками растянутых образцов можно предположить, что значительное увеличение сопротивления деформированию при больших степенях растяжения связано с локализацией деформации, т.е. появление «плавающих» шеек в рабочей части образца приводит к увеличению скорости деформации в этом участке, что свою очередь вызывает скоростное упрочнение материала. Но в то же время эффект наблюдается вблизи оптимальных режимов СП, что позволяет также говорить о возможном влиянии изменения фазово-структурного состояния и/или механизмов СПД.

Полученные экспериментальные результаты согласуются с теоретическими концепциями, выдвинутыми в работах В.Е. Панина с сотрудниками, в которых деформируемое твердое тело представляется как многоуровневая нелинейная иерархически организованная система $[7,8]$.

\section{Выводы}

1. Разработанный в данной статье метод фотосъемки позволяет определить течение истинных напряжений при сверхпластической деформации материалов и их стадийность.

2. Выявлен эффект аномального повышения истинных напряжений течения в предразрушительном этапе деформации, наиболее выраженный при оптимальных режимах СП.

3. Установлено, что в процессе сверхпластической деформации сплава $\mathrm{Co}-\mathrm{Ni}-\mathrm{Nb}$ идет коагуляция и рост вторичной фазы.

Работа выполнена при финансовой поддержке АО «Центр международных программ» РК и грантовой темы № 278 Комитета науки Министерства образования и науки РК.

\section{Список литературы}

[1] Hosford W.F. Mechanical Behavior of Materials. Cambridge University Press. Cambridge. UK, (2005) - 425 .

[2] Pilling J., Ridley N. Superplasticity in crystalline solids. The Institute of Metals (1989) 214.

[3] Ерболатулы Д., Скаков М.К., Гребнева В.С. и др. // Вестник КазНУ. Серия физическая. 2005. №1(19). С. 77-82.

[4] Новиков И.И., Портной В.К. Сверхпластичность сплавов с ультрамелким зерном. М.: Металлургия, 1981. $167 \mathrm{c}$.

[5] Золоторевский В.С. Механические испытания и свойства металлов. М.: Металлургия, 1974. $304 \mathrm{c}$.

$$
-809-
$$


[6] Кайбышев О.А. Сверхпластичность промышленных сплавов. М.: Металлургия, 1984. $264 \mathrm{c}$.

[7] Панин В.Е., Егорушкин В.Е. // Физическая мезомеханика. 2014. № 16 (3). С. 7-26.

[8] Головнев И.Ф., Головнева Е.И., Мержиевский Л.А. Фомин В.М. // Физическая мезомеханика. 2014. №16 (3). С. 35-43.

[9] Пресняков А.А., Аубакирова Р.К. Сверхпластичность металлических материалов. Алматы, 1982.235 с.

[10] Пресняков А.А., Аубакирова Р.К., Бакашева А.У., Хомов С.Н. // Известия МОН РК, НАН РК. Серия физико-математическая. 2001. №2. С. 88. 